\title{
A Novel New Energy Power Transmission System and Its Coordinate Control Strategy
}

\author{
WANG $\mathrm{Xi}^{1, \mathrm{a}}, \mathrm{CHEN}$ Gang ${ }^{1, \mathrm{~b}}, \mathrm{HU}$ Pengfei ${ }^{1, \mathrm{c}}$ \\ ${ }^{1}$ Sichuan Electrical Engineering Research Institute of State Grid, Chengdu 610000, China \\ aemail:wangxi_55@sina.cn, bemail:gangchen_thu@163.com, 'email:hupengfei01@163.com
}

Keywords: LCC-HVDC; VSC-HVDC; power balance; supplementary controller

\begin{abstract}
A novel series-structure power transmission system connected by LCC-HVDC (line commuted converter based High Voltage Direct Current) and VSC-HVDC (voltage sourced converter based High Voltage Direct Current) is studied. The coordinate supplementary control of LCC-HVDC and VSC-HVDC is proposed to deal with the possible power imbalance at LCC-HVDC sending end. The active power supplementary controller can effectively enhance the active power balance capacity at sending area. Besides, the reactive power supplementary controller of VSC-HVDC is designed to deal with the voltage fluctuation caused by LCC-HVDC active power supplementary control. Simulation results show that the cooperation between active and reactive power supplementary controller effectively enhances the power balance ability of the system.
\end{abstract}

\section{Introduction}

High-voltage Direct Current transmission technology can be divided into conventional LCC-HVDC (line commuted the converter -based High Voltage Direct Current) and VSC - HVDC (Voltage sourced converter -based High Voltage Direct Current) based on using the type of power electronic converter. At present, conventional HVDC technology is relatively mature, it is mainly used for large-scale power transmission over a long distance or asynchronous network since it has the characters of the high voltage level and large conveying capacity. Compared with LCC-HVDC, VSC-HVDC has more flexible control feathers like active power and reactive power independent control, and it has better dynamic voltage support ability for ac power grid. But the transmission capacity is relatively small and the voltage level is low compared with LCC-HVDC. So VSC-HVDC is usually used in interconnection between new energy power station such as wind farm and the main grid [1,2].

Because of the different characteristics, a novel series-structure power transmission system connected by LCC-HVDC and VSC-HVDC is very likely to be used in large new energy power base, since the power base need VSC-HVDC to collect the power and the LCC-HVDC to transmit the power to remote areas. Along with LCC-HVDC and VSC-HVDC hybrid transmission system, many documents has studied the operating characteristic of the two dc system, the influence between each other, and the coordinated failure recovery strategy[3-6]. These studies are mainly focus on the parallel-structure transmission system between LCC-HVDC and VSC-HVDC. In the paper, a novel series-structure power transmission system connected by LCC-HVDC and VSC-HVDC is analyzed.

\section{The structure and characteristics of the studied power transmission system}

For the convenience of the study, the studied series-structure power transmission system connected by LCC-HVDC and VSC-HVDC is simplified as shown in Fig.1. The VSC-HVDC is used to interconnect the new energy power station and the main grid since it has better controllability, while the LCC-HVDC is used to transmit power to remote area since it has large transmission capacity. 


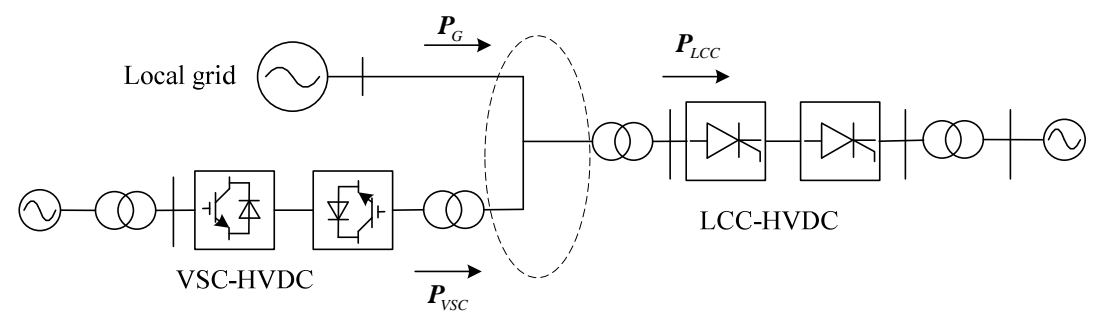

Fig. 1 Series-structure transmission system connected by LCC-HVDC and VSC-HVDC The studied power transmission system has the following characters

1) Power flow. The transmission power of VSC-HVDC and the power of local power grid is the power source of LCC-HVDC, the power flow is shown as fig.1.

2) The strength of local AC grid. The studied system is located in new energy power base which is usually in remote areas, so we consider the strength of local grid is weak.

3) Location. The transmission power of VSC-HVDC is mainly used to transmit by LCC-HVDC, so the inverter of VSC-HVDC and the rectifier of LCC-HVDC is near.

\section{The supplementary active power controller of VSC-HVDC and LCC-HVDC}

In normal condition, the control strategy of the two dc transmission system are

1) For LCC-HVDC, Constant current control and constant extinction angle control is used in the rectifier and inverter, respectively.

2) For VSC-HVDC, Constant active power control and constant ac voltage control is used in the rectifier, constant reactive power control and constant dc voltage control is used in the inverter.

If we only use the above conventional control strategy in HVDC control, the power balance in area 1 shown in fig. 1 is depends on the local weak ac system. We are know that the new energy output randomness is strong, only relying on the regulation of weak ac power grid is difficult to guarantee the security of the system. Based on this, we firstly proposed an active power supplementary control strategy of LCC-HVDC and VSC-HVDC to improve the power balance ability of area 1.

Ignore the power transmission losses, according to the balance of power

$P_{L C C}=P_{V S C}+P_{G}$

where $\mathrm{P}_{L C C}$ and $\mathrm{P}_{V S C}$ is the transmission power of LCC-HVDC and VSC-HVDC, $\mathrm{P}_{G}$ is the power of local ac grid transmit into area 1 . With HVDC conventional controller, $\mathrm{P}_{L C C}$ and $\mathrm{P}_{V S C}$ is constant. The imbalance power in area 1 can only be balanced by the power of local grid $\mathrm{P}_{G}$, since the strength of local grid is weak, the frequency fluctuation of the local grid may destroy the stability of the system. To improve the power balance ability, the following supplementary controller is designed shown as Fig.2.

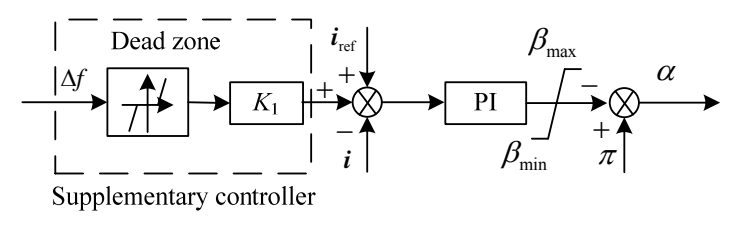

(a) LCC-HVDC

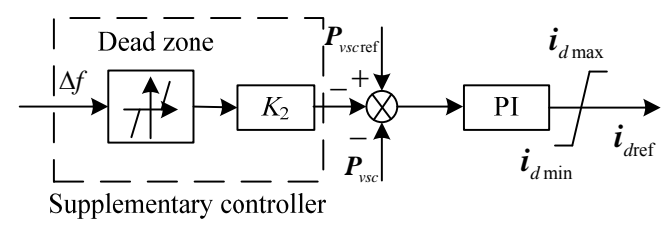

(b)VSC-HVDC

Fig. 2 Active power supplementary controller

The output of the supplementary controller is added to the constant current controller in LCC-HVDC and constant active power controller in VSC-HVDC, respectively. In the supplementary controller, $\Delta f$ is the frequency difference in area $1, K_{1}$ and $K_{2}$ are the control parameters. The dead zone is set to avoid the frequent action of the supplementary controller. The active power-frequency characteristic curve is no longer constant, which is shown as Fig.3 


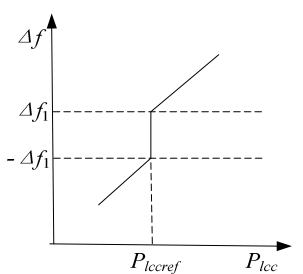

(a) LCC-HVDC

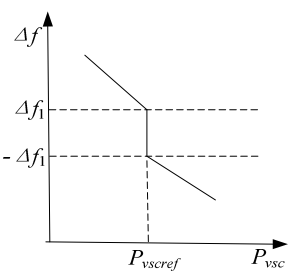

(b) VSC-HVDC

Fig. 3 Active power-frequency character with supplementary controller

When supplementary controllers act, the imbalance power in area 1 can be shared by local grid, LCC-HVDC and VSC-HVDC, which can be expressed as

$$
\begin{aligned}
\Delta P & =\Delta P_{G}+\Delta P_{v S C}-\Delta P_{L C C} \\
& =-K_{G} \Delta f-K_{l d c} \Delta f-K_{v d c} \Delta f \\
& =-\left(K_{G}+K_{l d c}+K_{v d c}\right) \Delta f
\end{aligned}
$$

where $\mathrm{K}_{\mathrm{G}}, \mathrm{K}_{l d c}, \mathrm{~K}_{v d c}$ is the frequency regulation coefficient of local grid, LCC-HVDC and VSC-HVDC, respectively. The value of the coefficient is equal to the absolute value of the ratio between active variation and frequency variation. It can be seen that the use of active power supplementary controller canreduce the frequency fluctuations which improves the stability of the system.

\section{The supplementary reactive power controller of VSC-HVDC and LCC-HVDC}

LCC-HVDC will consume much of reactive power in order to transmit active power. The consumed capacity of reactive power is $40-60 \%$ capacity of active power. The active power is mainly provided by the Reactive power compensator and filter, which means when the transmitted active power of LCC-HVDC is changed, the reactive power compensator has to be properly cut and equipped. While this is a kind of discontinuous voltage modulation, cutting reactive power compensator not only affect the service life of devices, but also cause strong voltage fluctuation. To avoid voltage fluctuation, we further propose a VSC-HVDC reactive power supplementary controller on the base of make full use of the good controllability of VSC-HVDC. The structure of the reactive supplementary controller is shown in Fig.4.

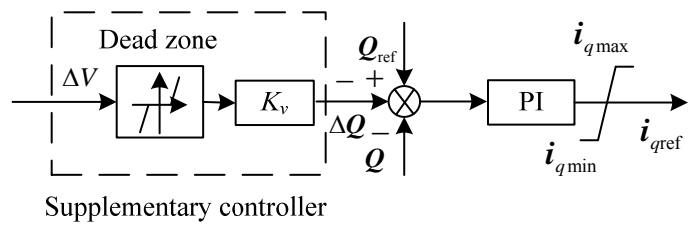

Fig. 4 Reactive power supplementary controller of VSC-HVDC

In Fig.4. $\Delta V=V-V_{\text {ref }}, V_{\text {ref }}$ and $V$ is the reference voltage and actual voltage of LCC-HVDC rectifier bus. $\mathrm{Kv}$ is the control parameter. Same as active supplementary controller, dead zone is set to avoid frequent action of the supplementary controller. With reactive supplementary controller, VSC-HVDC add reactive power output to stabilize the bus voltage of LCC-HVDC rectifier, when the actual voltage is lower than reference voltage. On the contrary, VSC-HVDC reduce reactive power output when bus voltage is higher than the reference. The reactive power-voltage character of VSC-HVDC with reactive power supplementary controller is shown as Fig 5.

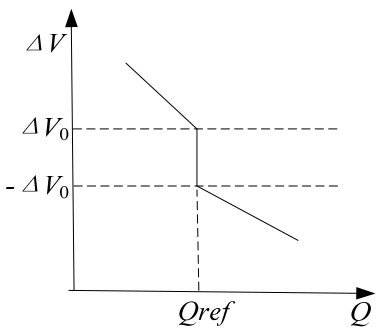

Fig. 5 Reactive power-voltage character of VSC-HVDC 


\section{Validation}

The test model shown as Fig.1 is set up in PSCAD/EMTDC to verify the effectiveness of the proposed supplementary control strategy. In the model, LCC-HVDC is under monopole operating mode, the local system is simulated by 7 generators.

Under normal condition, ac voltage is $345 \mathrm{kV}$, frequency is $50 \mathrm{~Hz}$, the transmission power of local grid, VSC-HVDC and LCC-HVDC is $600 \mathrm{MW}, 400 \mathrm{MW}$ and $1000 \mathrm{MW}$, respectively. To validate the proposed control strategy, we set the transmission power of VSC-HVDC change from 400MW to $250 \mathrm{MW}$ at $2 \mathrm{~s}$ to simulate the new energy output fluctuations. The simulation result without LCC-HVDC and VSC-HVDC supplementary controller is shown as Fig.6(a). We can see that after VSC-HVDC transmission power reduction, there is a $150 \mathrm{MW}$ power gap in area 1 . Since there is no VSC-HVDC and LCC-HVDC supplementary controller, local generators have to increase power output so that system frequency keep decreasing until the system lose stability.

To keep the system stable, LCC-HVDC and VSC-HVDC supplementary controller is added, the simulation result is shown in Fig 6(b).

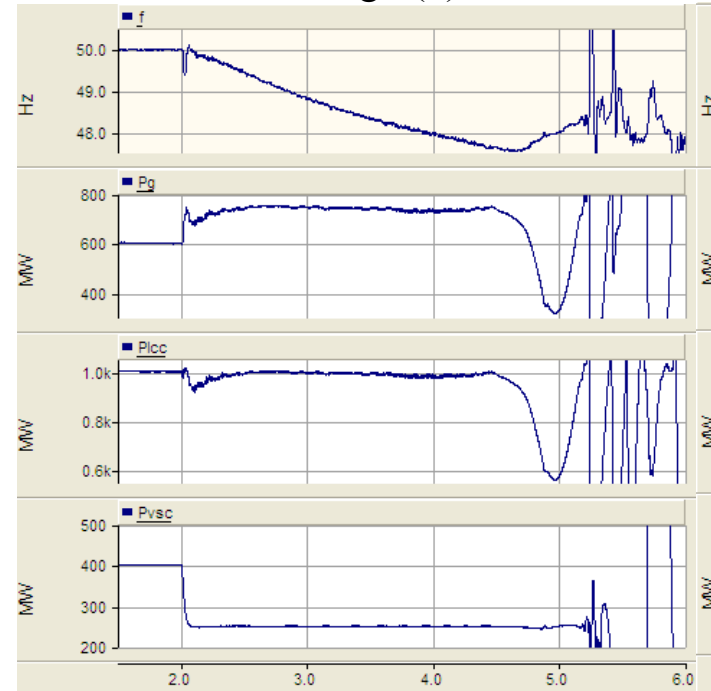

(a)without supplementary controller

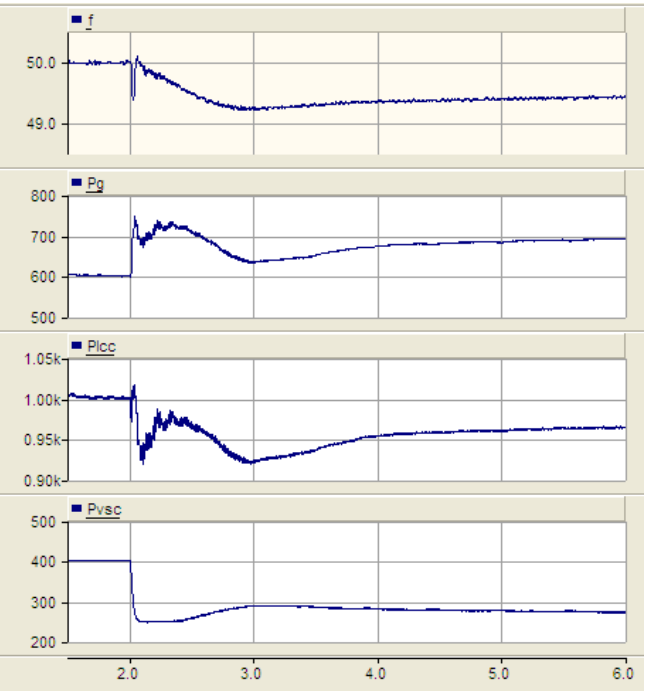

(b)with supplementary controller

Fig. 6 Simulation result

It can be seen that the system keeps stable at a frequency of $49.43 \mathrm{~Hz}$. When transmission power changed, LCC-HVDC and VSC-HVDC change transmission power along with frequency changes. To stabilize the system, LCC-HVDC changes transmission power from $1000 \mathrm{MW}$ to $965 \mathrm{MW}$, VSC-HVDC changes transmission power from $250 \mathrm{MW}$ to $275.5 \mathrm{MW}$. The imbalance power is shared by local grid, LCC-HVDC and VSC-HVDC rather than local grid only, so that the system keeps stable eventually.

In terms of reactive power and bus voltage, if no reactive supplementary controller is used, the output reactive power of VSC-HVDCkeep constant zero. The simulation result is given in Fig.7.We can see that the voltage bus of LCC-HVDC will have a big fluctuation if we suppose reactive power of LCC-HVDC keep constant.

To reduce the fluctuation of LCC-HVDC bus voltage, the proposed reactive power supplementary controller is used. Simulation results is shown in Fig.7. 


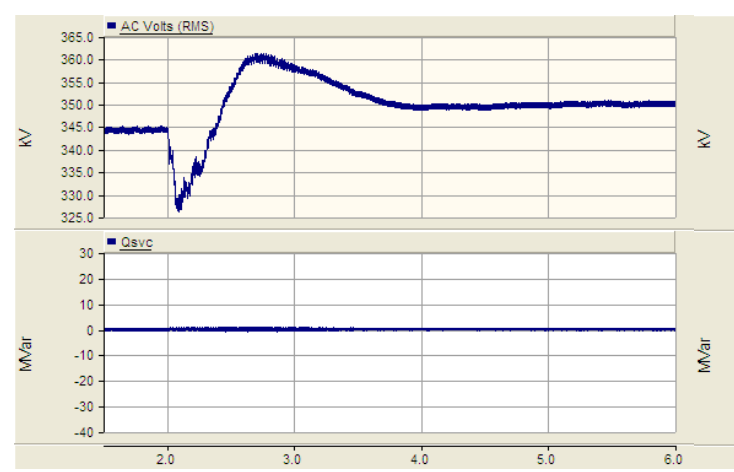

(a)without supplementary controller

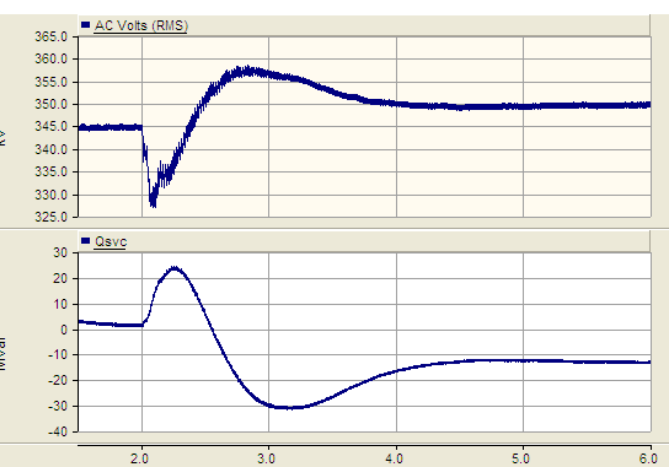

(b)with supplementary controller

Fig. 7 Simulation result with/without VSC-HVDC reactive power supplementary controller

It can be seen when the bus voltage of LCC-HVDC decrease, VSC-HVDC output more reactive power to stabilize the bus voltage. On the other hand, when the bus voltage gets higher, VSC-HVDC absorb the extra reactive power. In this way, the stability of LCC-HVDC bus voltage is enhanced by VSC-HVDC reactive power supplementary controller without any action of its own reactive power compensator.

\section{Conclusion}

In this paper, a novel series-structure transmission system connected by LCC-HVDC and VSC-HVDC is proposed and the coordination control strategy of the system is designed. With active power supplementary controller, the frequency stability of the studied ac system can be enhanced, and the power balance ability of system is improved. Furthermore, the VSC-HVDC reactive power supplementary controller is designed to keep LCC-HVDC bus voltage stable when it participates in active power balance. In this way, reactive power supplementary controller play the role of STATCOM. With the proposed active and reactive power supplementary controller, the system stability can be effectively improved.

\section{Reference}

[1] E. W. Kimbark. Direct Current Transmission[M]. John Wiley \& Sons, Inc., 1971.

[2] M. Szechtman, T. Wess, C. V. Thio. A Benchmark Model for HVDC System Studies[C]. International Conference on AC and DC Power Transmission, 1991, 374-378.

[3] Chunyi, G. and Z. Chengyong. Supply of an Entirely Passive AC Network Through a Double-Infeed HVDC System[J]. IEEE Transactions on Power Electronics, 2010, 25 (11): 2835-2841.

[4] Chunyi Guo, Yi Zhang, A. M. Gole, et al. Analysis of Dual-Infeed HVDC with LCC-HVDC and VSC-HVDC[J]. IEEE Transactions on Power Delivery, 2012, 27 (3): 1529-1537.

[5] ZHAO Cheng-yong, SUN Ying, LI Guang-kai. Control Strategy of VSC-HVDC in Dual-infeed HVDC Systems[J]. Proceedings of the CSEE，2008，28(7): 97-103.

[6] ZHAO Chengyong, HU Dongliang, LI Guangkai. Analysis on Reactive Power Regulation in New Type Double-infeed HVDC System[J]. Automation of Electric Power Systems, 2008, 32(21):51-55 . 